\title{
Exact first-passage time distributions for three random diffusivity models
}

\author{
Denis S. Grebenkov ${ }^{\dagger, \ddagger}$, Vittoria Sposini ${ }^{\ddagger, b}$, Ralf Metzler ${ }^{\ddagger}$, Gleb \\ Oshanin $\sharp$, and Flavio Seno \\ † Laboratoire de Physique de la Matière Condensée (UMR 7643), CNRS - Ecole \\ Polytechnique, IP Paris, 91128 Palaiseau, France \\ $\ddagger$ Institute for Physics and Astronomy, University of Potsdam, 14476 Potsdam-Golm, \\ Germany \\ b Basque Center for Applied Mathematics, 48009 Bilbao, Spain \\ \# Sorbonne Université, CNRS, Laboratoire de Physique Théorique de la Matière \\ Condensée (UMR 7600), 4 Place Jussieu, 75252 Paris Cedex 05, France \\ I INFN, Padova Section and Department of Physics and Astronomy "Galileo \\ Galilei", University of Padova, 35131 Padova, Italy \\ E-mail: Corresponding author: denis.grebenkov@polytechnique.edu
}

\begin{abstract}
We study the extremal properties of a stochastic process $x_{t}$ defined by a Langevin equation $\dot{x}_{t}=\sqrt{2 D_{0} V\left(B_{t}\right)} \xi_{t}$, where $\xi_{t}$ is a Gaussian white noise with zero mean, $D_{0}$ is a constant scale factor, and $V\left(B_{t}\right)$ is a stochastic "diffusivity" (noise strength), which itself is a functional of independent Brownian motion $B_{t}$. We derive exact, compact expressions in one and three dimensions for the probability density functions (PDFs) of the first passage time (FPT) $t$ from a fixed location $x_{0}$ to the origin for three different realisations of the stochastic diffusivity: a cut-off case $V\left(B_{t}\right)=\Theta\left(B_{t}\right)$ (Model I), where $\Theta(z)$ is the Heaviside theta function; a Geometric Brownian Motion $V\left(B_{t}\right)=\exp \left(B_{t}\right)$ (Model II); and a case with $V\left(B_{t}\right)=B_{t}^{2}$ (Model III). We realise that, rather surprisingly, the FPT PDF has exactly the Lévy-Smirnov form (specific for standard Brownian motion) for Model II, which concurrently exhibits a strongly anomalous diffusion. For Models I and III either the left or right tails (or both) have a different functional dependence on time as compared to the Lévy-Smirnov density. In all cases, the PDFs are broad such that already the first moment does not exist. Similar results are obtained in three dimensions for the FPT PDF to an absorbing spherical target.
\end{abstract}

Keywords: diffusing diffusivity, first passage time, Lévy-Smirnov density

PACS numbers: 02.50.-r, 05.40.-a, 02.70.Rr, 05.10.Gg

\section{Introduction}

There is strong experimental evidence that in some complex environments the observation of a "diffusive" behaviour, i.e., of a mean-squared displacement growing linearly with time $t$ in the form $\overline{x_{t}^{2}} \sim t$ does not necessarily imply that the position probability density function $(\mathrm{PDF}) P(x, t)$ of finding a particle at position $x$ at time $t$ 
is Gaussian. In fact, significant departures from a Gaussian form have been reported, with $P(x, t)$ having cusp-like shapes in the vicinity of $x=0$, and/or exhibiting nonGaussian tails. Such a behaviour was observed, e.g., for the motion of micron-sized beads along nanotubes or in entangled polymer networks [1,2], in colloidal suspensions [3] or suspensions of swimming microorganisms [4], dynamics of tracers in arrays of nanoposts [5], transport at fluid interfaces [6-8], as well as for the motion of nematodes [9]. Even more complicated non-Gaussian distributions were observed in Dictyostelium discoideum cell motion [10,11] and protein-crowded lipid bilayer membranes [12,13]. An apparent deviation from Gaussian forms was evidenced in numerical simulations of particles undergoing a polymerisation process [14], which is known to be anomalous in the nonStokesian case [15].

One increasingly popular line of thought concerning the origins of such nonGaussian diffusion advocates a picture based on the overdamped Langevin equation

$$
\frac{d x_{t}}{d t}=\sqrt{2 D_{t}} \xi_{t}
$$

in which $\xi_{t}$ is a usual white noise with zero mean and covariance $\overline{\xi_{t} \xi_{t^{\prime}}}=\delta\left(t-t^{\prime}\right)$, while the diffusivity $D_{t}$ is an independent stochastic process which captures in a heuristic fashion all possible dynamical constraints, local stimuli and interactions that a particle may experience while moving in a heterogeneous complex environment. We note that this overdamped formulation is appropriate for the description of typical tracer particles in a liquid environment [16,17], e.g., of submicron tracer beads or fluorescently labelled macromolecules in living cells. For these systems deterministic forces such as gravity are typically also irrelevant.

In the pioneering work [18] Chubinsky and Slater put forth such a random diffusivity concept for dynamics in heterogeneous systems for which they coined the notion "diffusing diffusivity". Concretely, they modelled the diffusivity as a Brownian particle in a gravitational field limited by a reflecting boundary condition at $D_{t}=0$ in order to guarantee positivity and stationarity of the $D_{t}$ dynamics. In subsequent analyses elucidating various aspects of the diffusing diffusivity model it was assumed that $D_{t}$ is a squared Ornstein-Uhlenbeck process [19-21]. Finally, [22] use a formulation directly including an Ornstein-Uhlenbeck process for $D_{t}$. All these models feature a stochastic diffusivity with bounded fluctuations around a mean value, with a finite correlation time. When the process is started with an equilibrated diffusivity distribution, the mean squared displacement has a constant effective amplitude at all times, in contrast to non-equilibrium initial conditions [23]. The probability density function (PDF) $P(x, t)$ of such a process is not a Gaussian function at intermediate times. $\ddagger$ Instead, $P(x, t)$ exhibits a transient cusp-like behaviour in the vicinity of the origin and has exponential

$\ddagger$ The crossover from non-Gaussian to Gaussian forms distinguishes the diffusing diffusivity models here from the superstatistical approach [24] employed originally in [1,2]. In the latter case the shape of the position PDF is permanently non-Gaussian. 
tails. $\S$ Further extensions of this basic model were discussed in [23, 25, 26]. We also mention recent models for non-Gaussian diffusion with Brownian scaling $\overline{x_{t}^{2}} \sim t$ based on extreme value statistics [27] and multimerisation of the diffusing molecule [14, 28]. Generalisations to anomalous diffusion of the form $\overline{x_{t}^{2}} \sim t^{\alpha}$ with $\alpha \in(0,2)$ in terms of long-range correlated, fractional Gaussian noise was recently discussed [29,30], as well as a fractional Brownian motion generalisation [31] of the Kärger switching-diffusivity model [32]. Finally, the role of quenched disorder is analysed in [33].

We note that the diffusing diffusivity process appeared earlier in the mathematical finance literature, where it is used for the modelling of stock price dynamics. Indeed, if we redefine $d x_{t} / d t$ in equation (1) as $d \ln \left(S_{t}\right) / d t$, we recover the celebrated BlackScholes equation [34] for the dynamics of an asset price $S_{t}$ with zero-constant trend and stochastic volatility $\sqrt{2 D_{t}}$. In this context, the choice of a squared Ornstein-Uhlenbeck process for $D_{t}$ corresponds to the Heston model of stochastic volatility [35]. The process $x_{t}$ in equation (1) thus has a wider appeal beyond the field of transport in complex heterogeneous media.

Several ad hoc diffusing diffusivity models in which the PDFs exhibit a nonGaussian behaviour for all times have been analysed recently [36] from a more general perspective, i.e., not constraining the analysis to Brownian motion with $\overline{x_{t}^{2}} \sim t$ only, but also extending it to anomalous diffusion. In reference [36], which focused mostly on power spectral densities of individual trajectories $x_{t}$ of such processes - a topic which attracted recent interest [37-40] - the corresponding position PDFs were also obtained explicitly [36]. It was demonstrated that their functional form is very sensitive to the precise choice of $D_{t}$. Indeed, depending on the choice of $D_{t}$, one encounters a very distinct behaviour in the long time limit: the central part of the PDF may be Gaussian or non-Gaussian, diverge as $|x| \rightarrow 0$, or remain bounded in this limit, and also the tails may assume Gaussian, exponential, log-normal, or even power-law forms.

The concept of first passage time (FPT) is fundamental for a given stochastic process, as it quantifies when the variable of interest crosses a given threshold for the first time [41-43]. This could be the moment in time when a diffusing test particle first reaches a given distance away from its starting point, or when a stock market first crosses a preset threshold value. The concept of first passage is central for the physical chemistry of chemical reactions of diffusing reactants, for biology to model how animals succeed in random searching for food, or for financial mathematics. More formally, the first passage can be studied on the basis of the diffusion equation corresponding of the specific diffusive process and by assuming absorbing boundary conditions in the position where the target or threshold is located. In addition, the domain geometry and the target properties (fully absorbing or partially reflecting) must be included in the study [44]. Different facets of extremal and first-passage properties of diffusing diffusivity models were scrutinised in [45-47]. Results from this analysis show that in general heterogeneity and dynamic disorder broaden the first-passage time density,

$\S$ Depending on the specific model and the spatial dimension the exponential may have a sub-dominant power-law prefactor [19-22]. 
increasing the likelihood of both short and long target location times. Thus, while on average the reaction kinetics is slowed down, some realisations perform a faster search, and this is sufficient to increase the activation speed in diffusion-limited reactions, which are dominated by the non-asymptotic part of the first passage time behaviour.

In what follows we focus on the first passage properties of three models of generalised diffusing diffusivity introduced in [36]. In these models particle dynamics obeys the Langevin equation (1) with $D_{t}=D_{0} V\left(B_{t}\right)$, where $D_{0}$ is a proportionality factorthe diffusion coefficient - while $V\left(B_{t}\right)$ is a (dimensionless) functional of independent Brownian motion $B_{t}$, with $V(z)$ being a prescribed, positive-defined function. We here use the definition of Brownian motion $B_{t}$ in terms of the stochastic integral $B_{t}=2 D_{B} \int_{0}^{t} d t^{\prime} \zeta_{t^{\prime}}$, where $\zeta_{t}$ represents another (additional to the white noise process $\xi_{t}$ in equation (1)) Gaussian, zero mean, $\delta$-correlated white noise, such that $B_{t=0}=0$, $\left\langle B_{t}\right\rangle=0$, and

$$
\left\langle B_{t} B_{t^{\prime}}\right\rangle=2 D_{B} \min \left(t, t^{\prime}\right)
$$

Note that $D_{B}$ is the diffusion coefficient of the Brownian motion $B_{t}$ driving the diffusing diffusivity and is different from $D_{0}$. The latter represents a dimensional scale factor that can be associated to the diffusion coefficient of the particle. Here and henceforth, the angular brackets denote averaging with respect to all possible realisations of the Brownian motion $B_{t}$, while the bar corresponds to averaging over realisations of the white noise process. We note parenthetically that the extremal properties of the Langevin dynamics subordinated to another Brownian motion have been actively studied in the last years within the context of the so-called run-and-tumble dynamics. In this experimentally-relevant situation, the force acting on the particle is a functional of the rotational Brownian motion (see e.g., reference [48]).

Specifically we concentrate on the FPTs from a fixed position $x_{0}>0$ to a perfectly reacting target placed at the origin and determine the full FPT PDF $H\left(t \mid x_{0}\right)$ for different choices of the Brownian motion functional $V\left(B_{t}\right)$. In this way we are able to vary the time-dependent randomness introduced into the diffusivity $D_{t}$ according to the different physical scenarios to be studied. In particular, following the models introduced in [36], we select three choices for $V\left(B_{t}\right)$ :

(I) $V\left(B_{t}\right)=\Theta\left(B_{t}\right)$, where $\Theta(z)$ is the Heaviside theta function such that $\Theta(z)=1$ for $z \geq 0$, and zero, otherwise;

(II) $V\left(B_{t}\right)=\exp \left(-B_{t} / a\right)$ with a scalar parameter $a$; and

(III) $V\left(B_{t}\right)=B_{t}^{2} / a^{2}$.

In Model I, which we call "cut-off Brownian motion" the process $x_{t}$ undergoes a standard Brownian motion with diffusion coefficient $D_{0}$ once $B_{t}>0$, and pauses for a random time at its current location when $B_{t}$ remains at negative values. Albeit the meansquared displacement of $x_{t}$ grows linearly in time in this model (see reference [36]), this is indeed a rather intricate process, in which a duration of the diffusive tours and of the pausing times have the same broad distribution. We note that this model represents an alternative to other standard processes describing waiting times and/or trapping events. 
One could think of, for instance, the comb model, in which a particle, while performing standard Brownian motion along one direction, gets stuck for a random time in branches perpendicular to the direction of the relevant diffusive motion [49-51].

In Model II the diffusivity $D_{t}$ follows so-called Geometric Brownian Motion, as does an asset price in the Black-Scholes model [34]. Note that here the dynamics of $x_{t}$ is not diffusive - the process progressively freezes when $B_{t}$ goes in the positive direction and accelerates when $B_{t}$ performs excursions in the negative direction. Overall the latter dominate and the mean-squared displacement exhibits a very fast (exponential) growth with time. Lastly, in Model III the process $x_{t}$ accelerates when $B_{t}$ goes away from the origin in either direction, and we are thus facing again a super-diffusive behaviour: the process $x_{t}$ in equation (1) shows a random ballistic growth with time. In a way, such a behaviour resembles the so-called "scaled" diffusion because for typical realisations of the process $B_{t}$ one has $\left|B_{t}\right| \sim \sqrt{t}$ and, hence, $x_{t}$ evolves in the presence of a random force

whose magnitude grows with time in proportion to $\sqrt{t}$. As shown in [36] the position PDF of this process is Gaussian around the origin and exponential in the tails. This can be compared to scaled Brownian motion, a Markovian process with time dependent diffusion coefficient $\mathscr{K}(t) \sim t^{\alpha-1}$ in the ballistic limit $\alpha \rightarrow 2$, whose position PDF stays Gaussian at all times [52]. Conversely, heterogenous diffusion processes with position dependent diffusion coefficient $\overline{\mathscr{K}}(x) \sim|x|^{\beta}$ in the limit $\beta=1$ are also ballistic but have an exponential position PDF (with subdominant power-law correction) at all times [53].

For all three models we derive exact compact expressions for the FPT PDF $H\left(t \mid x_{0}\right)$ in one dimension and also evaluate their forms for the three-dimensional case, which thus generalise the known results for a standard Brownian motion. We note that for a standard Brownian motion in two dimensions, the FPT PDF is known only in form of an inverse Laplace transform and via an integral representation [54,55]. Although an analogous expression for diffusing diffusivity models under study can be found rather directly from our general results (see below), we do not present such an analysis here because the resulting expressions appear to be rather cumbersome. We remark that, in general, the exact FPT PDFs are known in closed-form only for a very limited number of situations (see, e.g., [41-43,56-58], compare [59,60] for a "simple" spherical system). Thus, our results provide novel and non-trivial examples of stochastic processes for which the full FPT PDF can be calculated exactly and appears to have a simple explicit form.

The paper is outlined as follows. In section 2 we present some general arguments relating the FPT PDF and the position $\mathrm{PDF} P(x, t)$ for the processes governed by equation (1). In section 3 we present our results for the three models under study. Finally, in section 4 we conclude with a brief summary of our results and an outlook.

\section{General setup}

A general approach for evaluating the FPT PDF for the diffusing diffusivity models in equation (1) was developed in [45] (see also [46,47]). In this approach, one takes 
the advantage of the statistical independence of thermal noise and of the stochastic diffusivity $D_{t}$. Qualitatively speaking, the thermal noise determines the statistics of the stochastic trajectories of the process $x_{t}$, whereas the diffusing diffusivity controls the "speed" at which the process runs along these trajectories. As a consequence, for a particle starting from $x_{0}$ at time 0 the FPT PDF to a target, $H\left(t \mid x_{0}\right)$, can be obtained via subordination [45] (see also [21]),

$$
H\left(t \mid x_{0}\right)=\int_{0}^{\infty} d T q(t ; T) H_{0}\left(T \mid x_{0}\right)
$$

where $H_{0}\left(T \mid x_{0}\right)$ is the FPT PDF to the same target for ordinary Brownian motion with a constant diffusivity $D_{0}$, and $q(t ; T)$ is the PDF of the first-crossing time $\tau$ of a level $T$ by the integrated diffusivity,

$$
\tau=\inf \left\{t>0: T_{t}>T\right\}, \quad T_{t}=\int_{0}^{t} d t^{\prime} D\left(t^{\prime}\right) .
$$

In other words, $T_{t} / D_{0}$ plays the role of a "stochastic internal time" of the process $x_{t}$, which relates it to ordinary diffusion. The PDF $q(t ; T)$ can be formally determined by inverting the identity [45]

$$
\int_{0}^{\infty} d T e^{-\lambda T} q(t ; T)=-\frac{\partial \Upsilon(t ; \lambda) / \partial t}{\lambda}
$$

where $\Upsilon(t ; \lambda)$ is the generating function of the integrated diffusivity,

$$
\Upsilon(t ; \lambda)=\left\langle\exp \left(-\lambda \int_{0}^{t} d t^{\prime} D\left(t^{\prime}\right)\right)\right\rangle=\left\langle\exp \left(-D_{0} \lambda \int_{0}^{t} d t^{\prime} V\left(B_{t^{\prime}}\right)\right)\right\rangle .
$$

In the case of a constant diffusivity, $V(z)=1$, one simply gets $\Upsilon(t ; \lambda)=\exp \left(-D_{0} \lambda t\right)$.

When the process $x_{t}$ is confined to a bounded Euclidean domain $\Omega \subset \mathbb{R}^{d}$, the FPT PDF $H\left(t \mid x_{0}\right)$ can be obtained via a spectral expansion over the eigenvalues $\lambda_{n}$ and eigenfunctions $u_{n}$ of the Laplace operator in which the conventional time-dependence via $e^{-D_{0} t \lambda_{n}}$ is replaced by $\left(-\partial \Upsilon\left(t ; \lambda_{n}\right) / \partial t\right)[45]$. In fact, substituting the spectral expansion for ordinary diffusion [41],

$$
H_{0}\left(T \mid x_{0}\right)=\sum_{n} \lambda_{n} e^{-T \lambda_{n}} u_{n}\left(x_{0}\right) \int_{\Omega} d x u_{n}(x),
$$

into equation (3), one gets with the aid of equation (5) that

$$
H\left(t \mid x_{0}\right)=\sum_{n}\left(-\partial_{t} \Upsilon\left(t ; \lambda_{n}\right)\right) u_{n}\left(x_{0}\right) \int_{\Omega} d x u_{n}(x) .
$$

In turn, the analysis is more subtle for unbounded domains as one can no longer rely on spectral expansions. 
In what follows we focus on two emblematic unbounded domains, for which the FPT probability density $H_{0}\left(T \mid x_{0}\right)$ for ordinary diffusion is known:

(i) $x_{t}$ evolving on a half-line $(0, \infty)$ with the starting point $x_{0}>0$ and a target placed at the origin, for which

$$
H_{0}\left(T \mid x_{0}\right)=\frac{x_{0} \exp \left(-x_{0}^{2} /(4 T)\right)}{\sqrt{4 \pi D_{0}\left(T / D_{0}\right)^{3}}}
$$

is the Lévy-Smirnov distribution (with $T=D_{0} t$ ). Substituting this function into equation (3), one gets [45]

$$
H\left(t \mid x_{0}\right)=\frac{2}{\pi} \int_{0}^{\infty} \frac{d k}{k} \sin \left(k x_{0}\right)\left(-\partial_{t} \Upsilon\left(t ; k^{2}\right)\right) .
$$

Note that the position PDF reads

$$
P\left(x, t \mid x_{0}\right)=\int_{0}^{\infty} \frac{d k}{\pi} \cos \left(k\left(x-x_{0}\right)\right) \Upsilon\left(t ; k^{2}\right),
$$

such that the two PDFs are related via $\partial P\left(0, t \mid x_{0}\right) / \partial t=2 \partial H\left(t \mid x_{0}\right) / \partial x_{0}$. This is specific to the half-line problem.

(ii) In the second case we consider the dynamics in a three-dimensional (3d) region outside of an absorbing sphere of radius $R$. In this case one has for ordinary diffusion

$$
H_{0}^{3 d}\left(T \mid x_{0}\right)=\frac{R \exp \left(-\left(\left|x_{0}\right|-R\right)^{2} /(4 T)\right)}{\sqrt{4 \pi D_{0}\left(T / D_{0}\right)^{3}}},
$$

for any starting point $x_{0} \in \mathbb{R}^{3}$ outside the target, i.e., with $\left|x_{0}\right|>R$. Comparing equations (10) and (12) one gets for any diffusing diffusivity process $D_{t}$ :

$$
H^{3 d}\left(t \mid x_{0}\right)=\frac{R}{\left|x_{0}\right|} H\left(t|| x_{0} \mid-R\right)
$$

with $H\left(t \mid x_{0}\right)$ given by equation (10). Note that the position PDF in this case

$$
P\left(x, t \mid x_{0}\right)=\frac{1}{2 \pi^{2}\left|x-x_{0}\right|} \int_{0}^{\infty} d k k \sin \left(k\left|x-x_{0}\right|\right) \Upsilon\left(t ; k^{2}\right) .
$$

We highlight that in an unbounded three-dimensional space some trajectories travel to infinity and never reach the target, such that the target survival probability reaches a non-zero value when time tends to infinity. This implies that the FPT PDF is not normalised with respect to the set of all possible trajectories $x_{t}$. In standard fashion, the PDF in equation (13) can be renormalised over the set of such trajectories which do reach the target up to time moment $t$.

Using these general results, we now obtain closed-formed expressions for the FPT PDF of models I, II, and III. 


\section{Results}

\subsection{Model I}

We first consider the functional form $V\left(B_{t}\right)=\Theta\left(B_{t}\right)$, for which the generating function of the integrated diffusivity can be straightforwardly determined by taking advantage of the celebrated results due to Kac [61] and Kac and Erdös [62]. In our notations, we have

$$
\Upsilon\left(t ; q^{2}\right)=\exp \left(-\frac{D_{0} q^{2} t}{2}\right) I_{0}\left(\frac{D_{0} q^{2} t}{2}\right),
$$

where $I_{0}(z)$ is the modified Bessel function of the first kind of order zero defined as [63]

$$
I_{0}(z)=\frac{1}{\pi} \int_{0}^{\pi} d \theta e^{z \cos \theta} \cos \theta
$$

Note that the inverse Laplace transform of the expression in equation (15) produces the celebrated Lévy arcsine law [64]. Curiously, this expression does not depend on the diffusion coefficient $D_{B}$ of Brownian motion $B_{t}$ driving the diffusing diffusivity.

Substituting expression (15) into equation (10) we get

$$
H\left(t \mid x_{0}\right)=\frac{x_{0}}{\sqrt{4 \pi^{3} D_{0} t^{3}}} \exp \left(-\frac{x_{0}^{2}}{8 D_{0} t}\right) K_{0}\left(\frac{x_{0}^{2}}{8 D_{0} t}\right),
$$

where $K_{0}(z)$ is the modified Bessel function of the second kind of order zero [63],

$$
K_{0}(z)=\int_{0}^{\infty} d t \cos (z \sinh t)
$$

For completeness we also provide the moment-generating function of the FPT $\mathcal{T}$,

$$
\overline{\langle\exp (-\lambda \mathcal{T})}\rangle=\frac{2}{\pi} \int_{x_{0}}^{\infty} d z K_{0}(z),
$$

where the integral can also be represented in terms of modified Struve functions [63].

Due to the presence of $K_{0}(z)$, the FPT PDF $H\left(t \mid x_{0}\right)$ is functionally different from the conventional Lévy-Smirnov probability density (9) - we denote it as $H^{(\mathrm{LS})}\left(t \mid x_{0}\right)$ and this difference manifests itself both in the left and right tails of the FPT PDF. At short times $t \ll x_{0}^{2} /\left(8 D_{0}\right)$, (i.e., for the left tail of the FPT PDF), one gets from equation (17)

$$
H\left(t \mid x_{0}\right) \simeq \frac{\exp \left(-x_{0}^{2} /\left(4 D_{0} t\right)\right)}{\pi t} \quad(t \rightarrow 0)
$$

meaning that the PDF acquires, due to the presence of $K_{0}(z)$, an additional factor $1 / \sqrt{t}$. As a consequence, $H_{0}^{(\mathrm{LS})}\left(t \mid x_{0}\right) / H\left(t \mid x_{0}\right) \simeq x_{0} / \sqrt{D_{0} t} \rightarrow \infty$ in this limit, implying that $H\left(t \mid x_{0}\right)$ vanishes faster than the Lévy-Smirnov density.

Conversely, at long times $t \gg x_{0}^{2} /\left(8 D_{0}\right)$ (i.e., for the right tail of the PDF), one has from equation (17)

$$
H\left(t \mid x_{0}\right) \simeq \frac{x_{0}}{\sqrt{4 \pi^{3} D_{0} t^{3}}}\left(\ln \left(\frac{16 D_{0} t}{x_{0}^{2}}\right)-\gamma\right),
$$




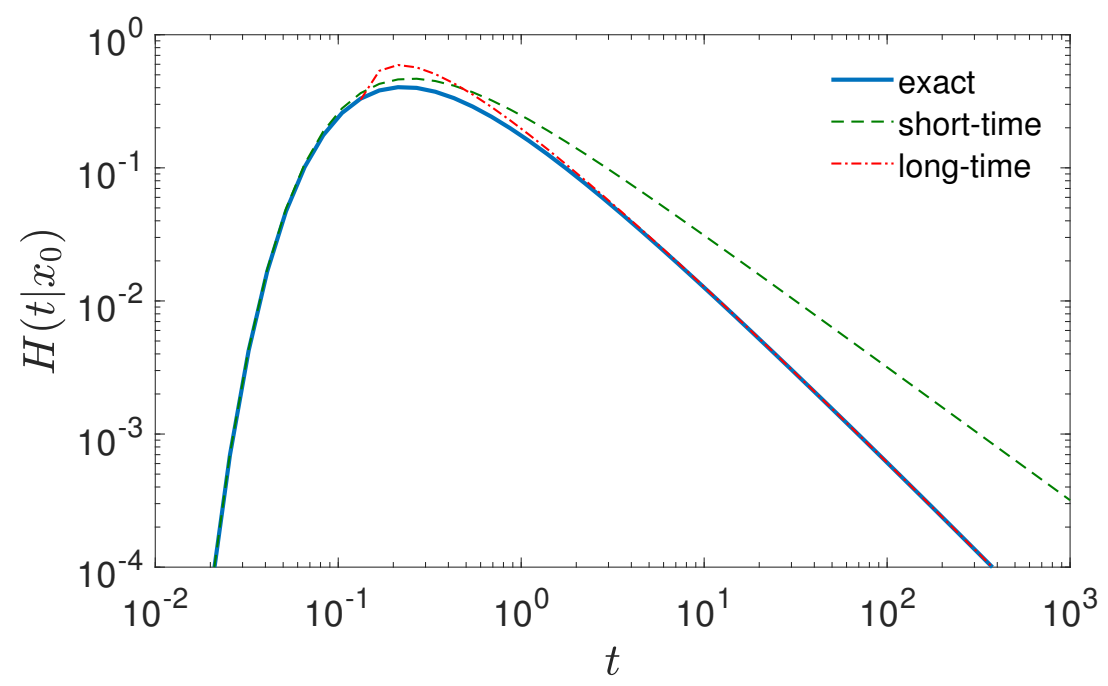

Figure 1. FPT PDF $H\left(t \mid x_{0}\right)$ of the diffusing diffusivity dynamics to the absorbing endpoint of the half-line $(0, \infty)$ for Model I. The thick solid line shows the exact form (17), while the thin dashed and dash-dotted lines present the short-time and long-time asymptotic relations (20) and (21), respectively. Here we set $x_{0}=1$ and $D_{0}=1$.

where $\gamma=0.5772$. is the Euler-Mascheroni constant [63]. Hence, in the long- $t$ limit the FPT PDF of Model I due to the additional logarithmic factor has a heavier tail than the Lévy-Smirnov density. Figure 1 illustrates the FPT PDF and its asymptotic behaviour.

We also note that the FPT PDF $H\left(t \mid x_{0}\right)$ resembles the free propagator of this diffusing diffusivity motion [36],

$$
P\left(x, t \mid x_{0}\right)=\frac{e^{-\left(x-x_{0}\right)^{2} /\left(8 D_{0} t\right)}}{\sqrt{4 \pi^{3} D t}} K_{0}\left(\left(x-x_{0}\right)^{2} /\left(8 D_{0} t\right)\right)=\frac{x_{0}}{t} H\left(t|| x-x_{0} \mid\right) .
$$

This curious effect follows from equations (10) and (11) and from the fact that $\Upsilon(t ; \lambda)$ for this model is only a function of $D_{0} t \lambda$.

\subsection{Model II}

For Model II we have $V\left(B_{t}\right)=\exp \left(-B_{t} / a\right)$ and the corresponding function $\Upsilon\left(t ; q^{2}\right)$ was evaluated within a different context in [65] - in fact, $\Upsilon\left(t ; q^{2}\right)$ is related to the momentgenerating function of the probability current in finite Sinai chains. Explicitly, $\Upsilon\left(t ; q^{2}\right)$ is defined by the Kontorovich-Lebedev transform

$$
\Upsilon\left(t ; q^{2}\right)=\frac{2}{\pi} \int_{0}^{\infty} d x \exp \left(-\frac{D_{B} t}{4 a^{2}} x^{2}\right) \cosh \left(\frac{\pi x}{2}\right) K_{i x}\left(2 a q \sqrt{\frac{D_{0}}{D_{B}}}\right),
$$

where $K_{i x}(z)$ is the modified Bessel function of the second kind with purely imaginary index [63]. We note that the exact forms of $\Upsilon(t ; \lambda)$ are also known for the case when $B_{t}$ experiences a constant drift $[66,67]$. Inserting this expression into equation (10) and 
performing the integrations we find that the FPT PDF of Model II is given explicitly by

$H\left(t \mid x_{0}\right)=\frac{a \operatorname{arcsinh}\left(x_{0} /\left(2 a \sqrt{D_{0} / D_{B}}\right)\right)}{\sqrt{\pi D_{B} t^{3}}} \exp \left(-\frac{a^{2} \operatorname{arcsinh}^{2}\left(\frac{x_{0}}{2 a \sqrt{D_{0} / D_{B}}}\right)}{D_{B} t}\right)$.

Remarkably, this is exactly the Lévy-Smirnov density of the form

$$
H\left(t \mid x_{0}\right)=\frac{X_{0}}{\sqrt{4 \pi D_{0} t^{3}}} \exp \left(-\frac{X_{0}^{2}}{4 D_{0} t}\right),
$$

with an effective starting point

$$
X_{0}=2 a \operatorname{arcsinh}\left(x_{0} /\left(2 a \sqrt{D_{0} / D_{B}}\right)\right) \text {, }
$$

dependent not only on $x_{0}$ but also on the diffusion coefficients $D_{0}$ and $D_{B}$ in a non-trivial way.

Expectedly, the moment-generating function for the FPT is simply given by a onesided stable law of the form

$$
\langle\overline{\exp (-\lambda \mathcal{T})}\rangle=\exp \left(-X_{0} \sqrt{\lambda / D_{0}}\right)
$$

as for Brownian motion.

\subsection{Model III}

For Model III we set $V\left(B_{t}\right)=B_{t}^{2} / a^{2}$. The function $\Upsilon\left(t ; q^{2}\right)$ can be calculated exactly by using the results of Cameron and Martin [68,69] (see also [70])

$$
\Upsilon\left(t ; q^{2}\right)=\frac{1}{\sqrt{\cosh (c q t)}},
$$

where $c=2 \sqrt{D_{B} D_{0} / a^{2}}$. Inserting this expression into equation (10) and performing the integral, we arrive at the rather unusual form of the FPT PDF

$$
H\left(t \mid x_{0}\right)=\frac{x_{0}}{\sqrt{2 \pi^{3}} c t^{2}} \Gamma\left(\frac{1}{4}+\frac{i x_{0}}{2 c t}\right) \Gamma\left(\frac{1}{4}-\frac{i x_{0}}{2 c t}\right),
$$

where $\Gamma(x)$ is the Gamma function. At short times, using the asymptotic formula $|\Gamma(a+i b)|^{2} \simeq 2 \pi e^{-\pi b} / \sqrt{b}$ as $b \rightarrow \infty$ for $a=1 / 4$, we get

$$
H\left(t \mid x_{0}\right) \simeq \frac{2 \sqrt{x_{0} / c}}{\sqrt{\pi t^{3}}} \exp \left(-\pi x_{0} /(2 c t)\right) .
$$

While the $t$-dependence of expression (30) is exactly the same as in the Lévy-Smirnov density, the dependence on $x_{0}$ is rather different, and also the PDF depends, through the constant $c$, on the diffusion coefficient $D_{B}$. In fact, setting

$$
X_{0}^{2}=\frac{2 \pi x_{0} D_{0}}{c}=\pi x_{0} a \sqrt{D_{0} / D_{B}}
$$




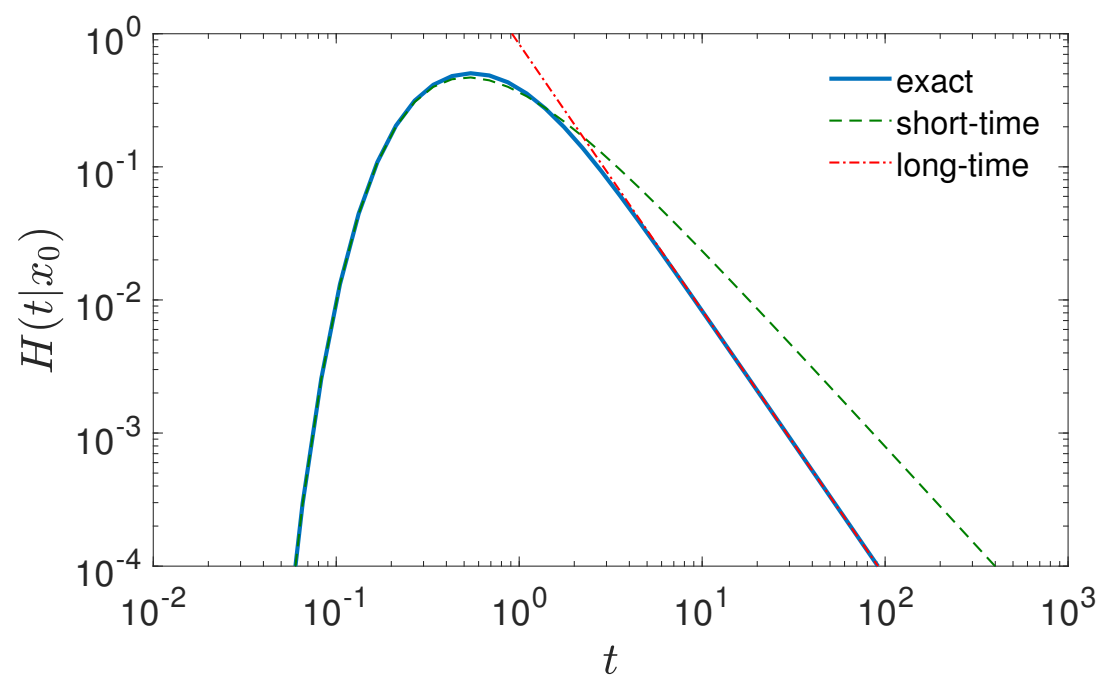

Figure 2. FPT PDF $H\left(t \mid x_{0}\right)$ to the absorbing endpoint of the half-line $(0, \infty)$ for Model III. The thick solid line shows the exact form (29) while the thin dashed and dash-dotted lines represent the short-time and long-time asymptotics (30) and (33), respectively. Here we set $x_{0}=1, D_{0}=1, D_{B}=1$, and $a=1$.

we can rewrite the short-time behaviour as

$$
H\left(t \mid x_{0}\right) \simeq \sqrt{8 / \pi} \frac{X_{0}}{\sqrt{4 \pi D_{0} t^{3}}} \exp \left(-X_{0}^{2} /\left(4 D_{0} t\right)\right),
$$

which is the Lévy-Smirnov distribution, except for the additional numerical factor $\sqrt{8 / \pi}$. At long times, the PDF exhibits the heavy tail

$$
H\left(t \mid x_{0}\right) \simeq \frac{x_{0}[\Gamma(1 / 4)]^{2}}{\sqrt{2 \pi^{3}} c} t^{-2},
$$

i.e., it decays faster than the Lévy-Smirnov distribution, but not fast enough to insure the existence of even the first moment. Figure 2 illustrates the FPT PDF $H\left(t \mid x_{0}\right)$ and its asymptotic behaviour.

Finally, the FPT PDF $H\left(t \mid x_{0}\right)$ is plotted for the three considered models in figure 3(a). In addition, we present the empirical histograms of the FPT generated by Monte Carlo simulations, observing excellent agreement. Moreover, we depict in figure 3(b) the FPT PDF $H\left(t \mid x_{0}\right)$ to an absorbing sphere of radius $R$ along with Monte Carlo simulations results. Details on Monte Carlo simulations are summarised in Appendix A.

\section{Conclusion}

First passage properties of a stochastic process are crucial for the quantification of secondary processes triggered by the arrival of the test particle to its target, such as chemical reactions or financial transactions. In financial market data the first passage dynamics with respect to a given, prescribed threshold value can immediately be 

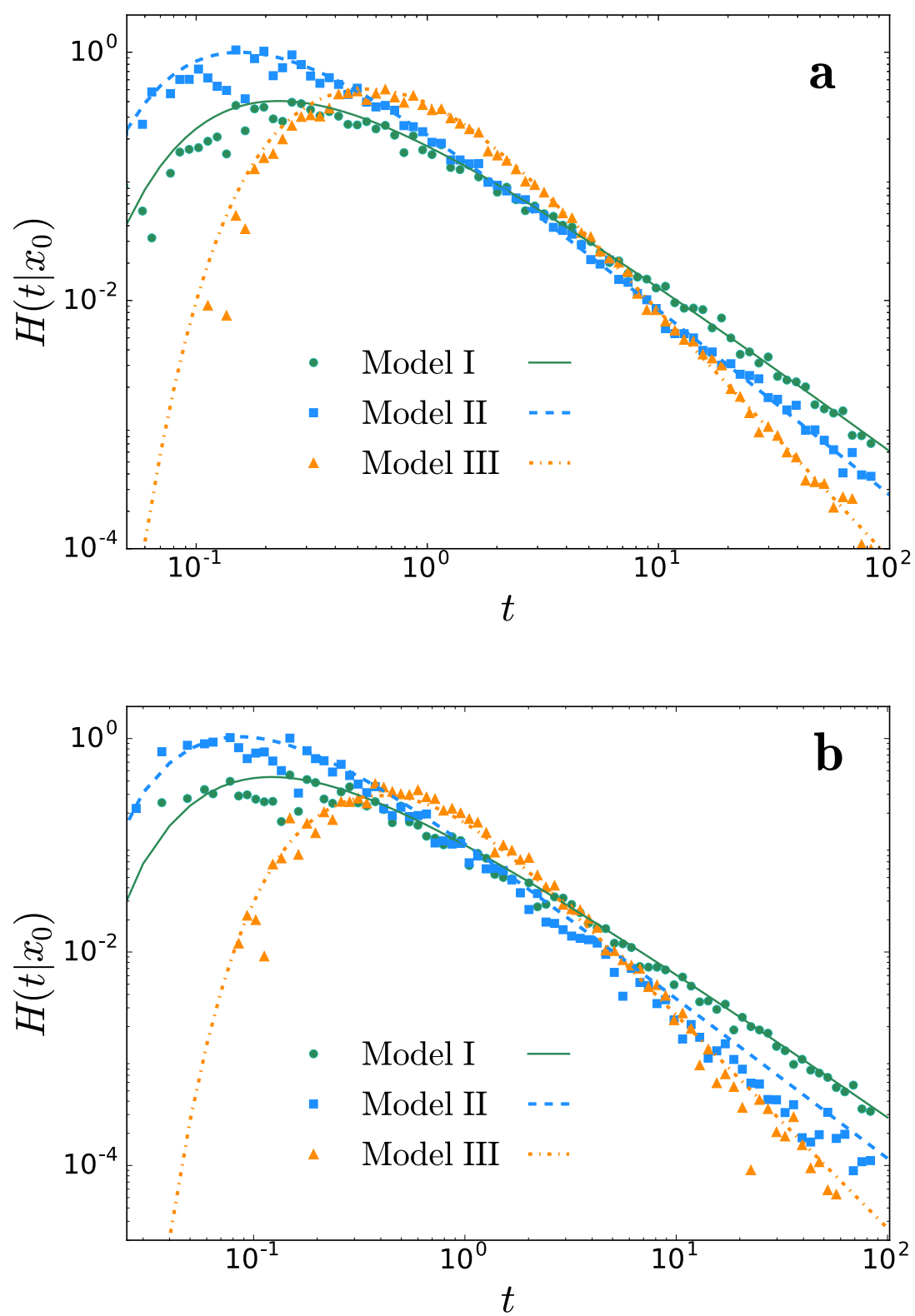

Figure 3. (a) Comparison of the FPT PDF $H\left(t \mid x_{0}\right)$ to the absorbing endpoint of the half-line $(0, \infty)$ for the three considered models. The solid lines represent the analytical results, while the symbols show the empirical histograms obtained from Monte Carlo simulations with $10^{4}$ runs. We set $x_{0}=1, D_{0}=1, D_{B}=1$, and $a=1$ (for Models II and III). (b) Similar comparison for the FPT PDF $H\left(t \mid x_{0}\right)$ to an absorbing sphere of radius $R=1$. The starting point is $\left|x_{0}\right|=\sqrt{3}$, while the other parameters are the same. 
studied. Similarly, in physical processes studied by simulations first passage properties are analysed in order to pinpoint the underlying physical process, see, e.g., the analysis in [?]. However, even in experimental systems such first passage properties are now routinely measured, by following fluorescently labelled, single particles by superresolution microscopes [?]. Detailed analytical predictions for the first passage behaviour of different stochastic processes are therefore needed for dedicated data analysis.

To this end, we studied the extremal properties of a stochastic process $x_{t}$ generated by the Langevin equation (1) with a stochastic diffusivity $V\left(B_{t}\right)$. The latter is taken to be a functional of an independent Brownian motion $B_{t}$. For three choices of the functional form of $V\left(B_{t}\right)$ we derived exact, compact expressions for the FPT PDF from a fixed initial location to the origin. Such distributions are known only for a very limited number of stochastic processes, and hence, our work provides novel examples of non-trivial processes for which this type of analysis can be carried out exactly. Similar results were obtained for the first passage time to an absorbing spherical target in three dimensions.

Following the recent reference [36], which revealed a universal large-frequency behaviour of spectral densities of individual trajectories $x_{t}$ for the three models studied here, one could expect the same short-time asymptotic behaviour of the FPT PDF for all these models, with a generic Lévy-Smirnov form. Indeed, in [36] it was shown that the spectral densities of individual realisations of $x_{t}$ decay as $1 / f^{2}$ when $f \rightarrow \infty$, i.e., exactly as the spectral density of standard Brownian motion. However, we realised here that the FPT PDF is of Lévy-Smirnov form (with an effective starting point, dependent on the diffusion coefficients $D_{B}$ and $D_{0}$ ) only for Model II, in which $V\left(B_{t}\right)$ is exponentially dependent on $B_{t}$, such that the process $x_{t}$ is strongly anomalous and its mean-squared displacement grows exponentially with time. In turn, for Model I with the cut-off Brownian motion $V\left(B_{t}\right)=\Theta\left(B_{t}\right)$, which exhibits a diffusive behaviour $\left\langle\overline{x_{t}^{2}}\right\rangle \propto t$, we observed essential departures from the Lévy-Smirnov form. We saw that the corresponding FPT PDF decays faster than the Lévy-Smirnov law in the limit $t \rightarrow 0$, and slower than the Lévy-Smirnov law in the limit $t \rightarrow \infty$. For Model III with the squared Brownian motion $V\left(B_{t}\right)=B_{t}^{2} / a^{2}$, the left tail of the FPT PDF has the LévySmirnov form with a renormalised starting point, while the right tail decays faster. In all models the distributions are broad such that even the first moment does not exist.

We conclude that the universal $1 / f^{2}$ decay of the spectral density does not distinguish between different diffusing diffusivity models. Indeed, as we discussed earlier, the white noise $\xi_{t}$ in the Langevin equation (1) determines the statistics of trajectories in space, whereas its amplitude, $\sqrt{2 D_{t}}$, can speed up or slow down the motion along each trajectory [45]. The spectral density is thus more sensitive to the spatial aspect of the dynamics, and its universal decay simply reflects that the statistics of the trajectories governed by the white noise is the same for all considered models. In turn, the FPT is also sensitive to the temporal aspect of the dynamics, i.e., to the "speed", at which the particle moves along the trajectory. This feature makes the spectral density and the 
first-passage time analysis techniques complementary.

It will be interesting to extend this analysis to other models for diffusion in heterogeneous media, in particular, when the driving noise is Gaussian but long-range correlated. For this case the behaviours of the mean squared displacement and the position PDF were recently considered in a superstatistical approach [71,72], in a generalised diffusing diffusivity picture $[29,30]$, as well as in terms of an intermittent two-state model of different particle mobility [31,73].

\section{Acknowledgments}

D.S.G. acknowledges a partial financial support from the Alexander von Humboldt Foundation through a Bessel Research Award. RM acknowledges the German Science Foundation (DFG grant ME 1535/7-1) and an Alexander von Humboldt Honorary Polish Research Scholarship from the Foundation for Polish Science (Fundacja na rzecz Nauki Polskiej, FNP). FS acknowledges financial support of the 191017 BIRD-PRD project of the Department of Physics and Astronomy of Padua University.

\section{Appendix A. Monte Carlo simulations}

Monte Carlo simulations are carried out to support analytical results. The Euler integration scheme is applied to simulate numerically the Langevin equation (1). For each realisation an independent Brownian motion run $B_{t}$ is generated to obtain the dimensionless random diffusivity through the specific functional $V\left(B_{t}\right)$ for each model. Note that in 3D, assuming an overall isotropy of the system, the same Brownian motion run is used to calculate the diffusivity along the three dimensions.

In order to implement the absorbing target, represented by a single point in $1 \mathrm{D}$ and by a sphere in 3D, the following procedure is used:

1D. The absorbing point is located at the origin and the initial position is $x_{0}>0$. At each step $t_{i}$ the algorithm checks whether $x_{t_{i}} \leq 0$; if the latter condition is true then the simulation is stopped and $t_{i}$ is stored as the first passage time for that trajectory, else the simulation carries on.

3D. The absorbing sphere is centred in the origin and has a radius $R$. The initial position $x_{0}$ is located outside of the absorbing sphere and will thus identify a sphere with initial radius $r_{0}=\left|x_{0}\right|>R$. At each step $t_{i}$ the algorithm checks whether $r_{t_{i}}=\left|x_{t_{i}}\right| \leq R$; if the latter condition is true the simulation is stopped and $t_{i}$ is stored as the first passage time for that trajectory, else the simulation carries on.

Note that, especially in the 3D case, there are trajectories that will diffuse away from the absorbing target and will (practically) never return to it. In order to overcome this issue a maximum simulation time $t_{\max }$ is fixed. This means that $t_{\max }$ will define a maximum for the first passage times obtained from simulations. For the results shown in this paper we set $t_{\max }=10^{2}$. 


\begin{tabular}{|l||ccc|ccc|}
\hline & \multicolumn{3}{|c|}{ 1D } & \multicolumn{3}{c|}{ 3D } \\
\hline & Model I & Model II & Model III & Model I & Model II & Model III \\
\hline$\epsilon$ & 0.2 & 0.2 & 0.3 & 0.2 & 0.3 & 0.3 \\
\hline
\end{tabular}

Table A1. Values for the estimator defined in equation (A.1) for the three models in both $1 \mathrm{D}$ and $3 \mathrm{D}$.

Histograms of the stored first passage times are created to estimate the first passage time distribution for each model, in both 1D and 3D. Results are shown in the main text, figure 3. One can see that there are small deviations of the histogram points from the analytical curves. Such deviations are due to statistical error. Indeed we can calculate the residuals, defined as the difference between the numerical and the analytical points, that is $H_{\text {num }}\left(t \mid x_{0}\right)-H_{\text {th }}\left(t \mid x_{0}\right)$, and plot them together with the statistical error bars. The latter are given by the square root of the counts within each bin-under the assumption that the counts follow a Poisson distribution. In figure A1 results of this analysis are shown. The residuals are always zero within the error bar. The only exceptions are given by the points in which no error bar is reported, corresponding to bins where there are no counts - nothing can be said about those points. In addition to this analysis the following quantity can be evaluated as a common estimator of the deviation from analytical curves,

$$
\epsilon=\frac{1}{N_{B}} \sum_{i=1}^{N_{B}} \frac{\left|H_{\mathrm{num}}\left(t_{i} \mid x_{0}\right)-H_{\mathrm{th}}\left(t_{i} \mid x_{0}\right)\right|}{H_{\mathrm{th}}\left(t_{i} \mid x_{0}\right)}
$$

where $N_{B}$ is the total number of bins. The results from equation (A.1) for the three models are reported in Table A1. The values vary between 0.2 and 0.3 , confirming our claim that numerical results and analytical predictions are in excellent agreement.

\section{References}

[1] B. Wang, J. Kuo, S. C. Bae, and S. Granick, When Brownian diffusion is not Gaussian, Nat. Mater. 11, 481 (2012).

[2] B. Wang, S. M. Anthony, S. C. Bae, and S. Granick, Anomalous yet Brownian, Proc. Natl. Acad. Sci. USA 106, 15160 (2009).

[3] J. Guan, B. Wang, and S. Granick, Single-molecule observation of long jumps in polymer adsorption, ACS Nano 8, 3331 (2014).

[4] K. C. Leptos, J. S. Guasto, J. P. Gollub, A. I. Pesci, and R. E. Goldstein, Dynamics of enhanced tracer diffusion in suspensions of swimming eukaryotic microorganisms, Phys. Rev. Lett. 103, 198103 (2009).

[5] K. He, F. B. Khorasani, S. T. Retterer, D. K. Tjomasn, J. C. Conrad, and R. Krishnamoorti, Diffusive dynamics of nanoparticles in arrays of nanoposts, ACS Nano 7, 5122 (2013).

[6] C. Xue, X. Zheng, K. Chen, Y. Tian, and G. Hu, Probing non-Gaussianity in confined diffusion of nanoparticles, J. Phys. Chem. Lett. 7, 514 (2016).

[7] D. Wang, R. Hu, M. J. Skaug, and D. Schwartz, Temporally anticorrelated motion of nanoparticles at a liquid interface, J. Phys. Chem. Lett. 6, 54 (2015).

[8] S. Dutta and J. Chakrabarti, Anomalous dynamical responses in a driven system, EPL 116, 38001 (2016). 

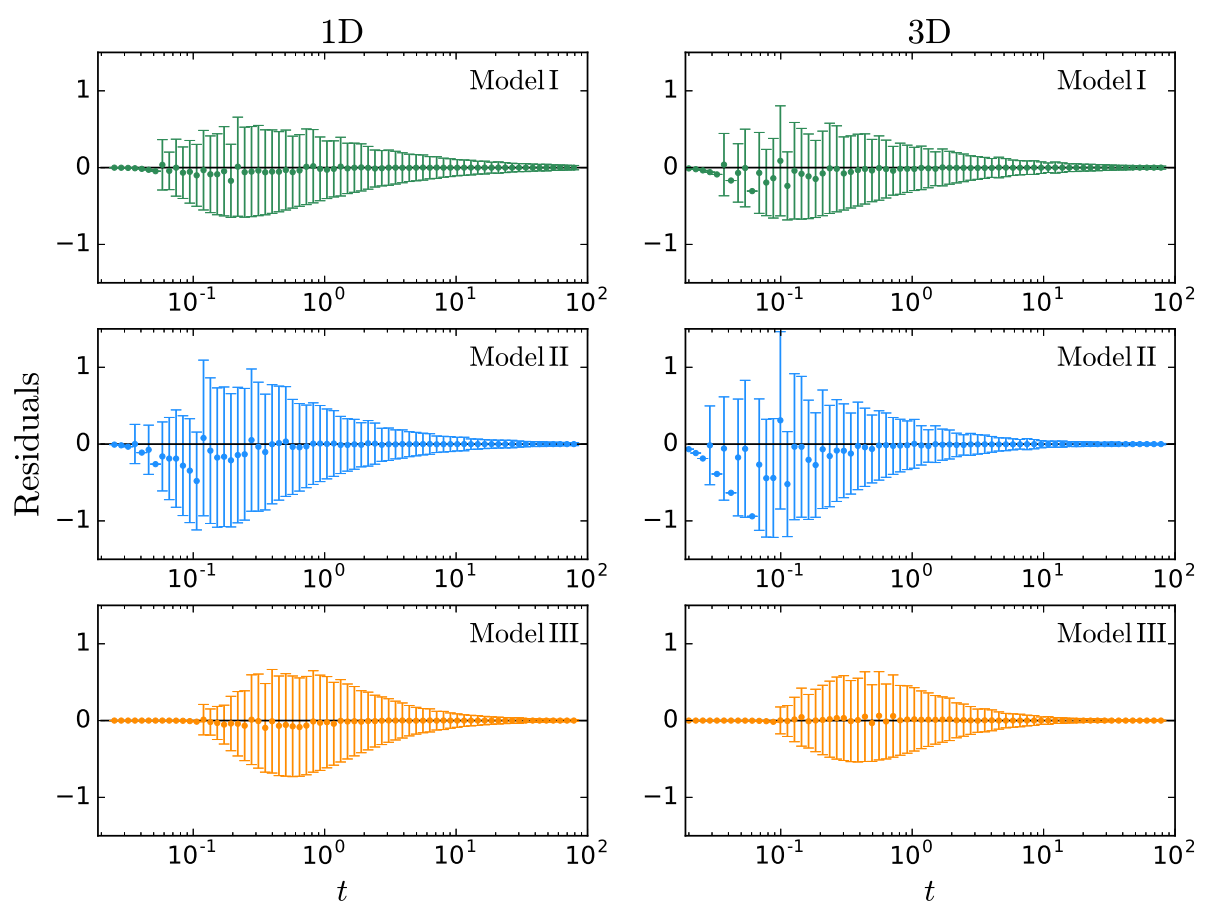

Figure A1. Residuals (dots) and error bars as an estimate of the agreement between the numerical histograms and the the corresponding analytical results for all three models in 1D (left panels) and 3D (right panels). The parameters are the same as in figure 3 .

[9] S. Hapca, J. W. Crawford, and I. M. Young, Anomalous diffusion of heterogeneous populations characterized by normal diffusion at the individual level, J. R. Soc. Interface 6, 111 (2009).

[10] P. Witzel, M. Götz, Y. Lanoiselée, T. Franosch, D. S. Grebenkov, and D. Heinrich, Heterogeneities Shape Passive Intracellular Transport, Biophys. J. 117, 203 (2019).

[11] A. G. Cherstvy, O. Nagel, C. Beta, and R. Metzler, Non-Gaussianity, population heterogeneity, and transient superdiffusion in the spreading dynamics of amoeboid cells, Phys. Chem. Chem. Phys. 20, 23034 (2018).

[12] J.-H. Jeon, M. Javanainen, H. Martinez-Seara, R. Metzler, and I. Vattulainen, Protein crowding in lipid bilayers gives rise to non-Gaussian anomalous lateral diffusion of phospholipids and proteins, Phys. Rev. X 6, 021006 (2016).

[13] W. He, H. Song, Y. Su, L. Geng, B. J. Ackerson, H. B. Peng, and P. Tong, Dynamic heterogeneity and non-Gaussian statistics for acetylcholine receptors on live cell membrane, Nat. Comm. 7, 11701 (2016).

[14] F. Baldovin, E. Orlandini, and F. Seno, Polymerization Induces Non-Gaussian Diffusion, Front. Phys. 7, 124 (2019).

[15] G. Oshanin and M. Moreau, Influence of transport limitations on the kinetics of homopolymerization reactions, J. Chem. Phys. 102, 2977 (1995).

[16] R. Huang, I. Chavez, K. M. Taute, Lukic, S. Jeney, M. G. Raizen, and E.-L. Florin, Direct observation of the full transition from ballistic to diffusive Brownian motion in a liquid, Nat. Phys. 7, 576-580 (2011).

[17] D. S. Grebenkov, M. Vahabi, E. Bertseva, L. Forro, and S. Jeney, Hydrodynamic and subdiffusive motion of tracers in a viscoelastic medium, Phys. Rev. E 88, 040701R (2013). 
[18] M. V. Chubynsky and G. W. Slater, Diffusing Diffusivity: A Model for Anomalous, yet Brownian, Diffusion, Phys. Rev. Lett. 113, 098302 (2014).

[19] R. Jain and K. L. Sebastian, Diffusion in a Crowded, Rearranging Environment, J. Phys. Chem. B 120, 3988 (2016).

[20] R. Jain and K. L. Sebastian, Diffusing diffusivity: survival in a crowded rearranging and bounded domain, J. Phys. Chem. B 120, 9215 (2016).

[21] A. V. Chechkin, F. Seno, R. Metzler, and I. M. Sokolov, Brownian yet Non-Gaussian Diffusion: From Superstatistics to Subordination of Diffusing Diffusivities, Phys. Rev. X 7, 021002 (2017).

[22] N. Tyagi and B. J. Cherayil, Non-Gaussian Brownian diffusion in dynamically disordered thermal environments, J. Phys. Chem. B 121, 7204 (2017).

[23] V. Sposini, A. V. Chechkin, F. Seno, G. Pagnini, and R. Metzler, Random diffusivity from stochastic equations: comparison of two models for Brownian yet non-Gaussian diffusion, New. J. Phys. 20, 043044 (2018).

[24] C. Beck and E. Cohen, Superstatistics, Physica A 322, 267 (2003).

[25] Y. Lanoiselée and D. S. Grebenkov, A model of non-Gaussian diffusion in heterogeneous media, J. Phys. A. 51, 145602 (2018).

[26] Y. Lanoiselée and D. S. Grebenkov, Non-Gaussian diffusion of mixed origins, J. Phys. A 52, 304001 (2019).

[27] E. Barkai and S. Burov, Packets of Diffusing Particles Exhibit Universal Exponential Tails, Phys. Rev. Lett. 124, 060603 (2020).

[28] M. Hidalgo-Soria and E. Barkai, Hitchhiker model for Laplace diffusion processes, Phys. Rev. E 102, 012109 (2020).

[29] W. Wang, F. Seno, I. M. Sokolov, A. V. Chechkin, and R. Metzler, Unexpected crossovers in correlated random-diffusivity processes, New J. Phys. 22, 083041 (2020).

[30] W. Wang, A. G. Cherstvy, A. V. Chechkin, S. Thapa, F. Seno, X. Liu, and R. Metzler, Fractional Brownian motion with random diffusivity: emerging residual nonergodicity below the correlation time, J. Phys. A., at press.

[31] A. Sabri, X. Xu, D. Krapf, and M. Weiss, Elucidating the Origin of Heterogeneous Anomalous Diffusion in the Cytoplasm of Mammalian Cells, E-print arXiv:1910.00102.

[32] J. Kärger, NMR self diffusion studies in heterogeneous systems, Adv. Coll. Interf. Sci. 23, 129 (1985).

[33] E. B. Postnikov, A. V. Chechkin, and I. M. Sokolov, Brownian yet non-Gaussian diffusion in heterogeneous media: from superstatistics to homogenization, New J. Phys. 22, 063046 (2020).

[34] F. Black and M. Scholes, The pricing of options and corporate liabilities, J. Polit. Econ. 81, 637 (1973).

[35] S. L. Heston, A Closed-Form Solution for Options with Stochastic Volatility with Applications to Bond and Currency Options, Rev. Financial Studies 6, 327 (1993).

[36] V. Sposini, D. S. Grebenkov, R. Metzler, G. Oshanin, and F. Seno, Universal spectral features of different classes of random diffusivity processes, New J. Phys. 22, 063056 (2020).

[37] D. Krapf, E. Marinari, R. Metzler, G. Oshanin, X. Xu and A. Squarcini, Power spectral density of a single Brownian trajectory: what one can and cannot learn from it, New J. Phys. 20, 023029 (2018).

[38] D. Krapf, N. Lukat, E. Marinari, R. Metzler, G. Oshanin, C. Selhuber-Unkel, A. Squarcini, L. Stadler, M. Weiss, and X. Xu, Spectral Content of a Single Non-Brownian Trajectory, Phys. Rev. X 9, 011019 (2019).

[39] V. Sposini, R. Metzler and G. Oshanin, Single-trajectory spectral analysis of scaled Brownian motion, New J. Phys. 21, 073043 (2019).

[40] S. N. Majumdar and G. Oshanin, Spectral content of fractional Brownian motion with stochastic reset, J. Phys. A: Math. Theor. 51, 435001 (2018).

[41] S. Redner, A Guide to First Passage Processes (Cambridge: Cambridge University Press, 2001).

[42] C. Mejía-Monasterio, G. Oshanin and G. Schehr, First passages for a search by a swarm of 
independent random searchers, Journal of Statistical Mechanics: Theory and Experiment (06), P06022 (2011).

[43] R. Metzler, G. Oshanin, and S. Redner (Eds.) First-Passage Phenomena and Their Applications (Singapore: World Scientific, 2014).

[44] D. S. Grebenkov, Paradigm Shift in Diffusion-Mediated Surface Phenomena, Phys. Rev. Lett. 125, $078102(2020)$.

[45] Y. Lanoiselée, N. Moutal, and D. S. Grebenkov, Diffusion-limited reactions in dynamic heterogeneous media, Nat. Commun. 9, 4398 (2018).

[46] V. Sposini, A. V. Chechkin, and R. Metzler, First passage statistics for diffusing diffusivity, J. Phys. A 52, 04LT01 (2019).

[47] D. S. Grebenkov, A unifying approach to first-passage time distributions in diffusing diffusivity and switching diffusion models, J. Phys. A 52, 174001 (2019).

[48] F. Mori, P. Le Doussal, S. N. Majumdar and G. Schehr, Universal survival probability for a ddimensional run-and-tumble particle, Phys. Rev. Lett. 124, 090603 (2020).

[49] V. E. Arkhincheev and E. M. Baskin, Anomalous diffusion and drift in a comb model of percolation clusters, Sov. Phys. JETP 73, 161 (1991).

[50] T. Sandev, A. Iomin, H. Kantz, R. Metzler and A. Chechkin, Comb model with slow and Ultraslow diffusion, Math. Model. Nat. Phenom. 11, 18 (2016).

[51] O. Bénichou, P. Illien, G. Oshanin, A. Sarracino and R. Voituriez, Diffusion and subdiffusion of interacting particles on comblike structures, Phys. Rev. Lett. 115, 220601 (2015).

[52] J-.H. Jeon, A. V. Chechkin, and R. Metzler, Scaled Brownian motion: a paradoxical process with a time dependent diffusivity for the description of anomalous diffusion, Phys. Chem. Chem. Phys. 16, 15811 (2014).

[53] A. G. Cherstvy, A. V. Chechkin, and R. Metzler, Anomalous diffusion and ergodicity breaking in heterogeneous diffusion processes, New J. Phys. 15, 083039 (2013).

[54] D. S. Grebenkov, R. Metzler, and G. Oshanin, Towards a full quantitative description of singlemolecule reaction kinetics in biological cells, Phys. Chem. Chem. Phys. 20, 16393-16401 (2018).

[55] D. S. Grebenkov, Statistics of boundary encounters by a particle diffusing outside a compact planar domain (accepted to J. Phys. A.: Math. Theor.)

[56] A. N. Borodin and P. Salminen, Handbook of Brownian Motion: Facts and Formulae (Birkhäuser Verlag, Basel-Boston-Berlin, 1996).

[57] A. J. Bray, S. Majumdar, and G. Schehr, Persistence and First-Passage Properties in Nonequilibrium Systems, Adv. Phys. 62, 225 (2013).

[58] O. Bénichou and R. Voituriez, From first-passage times of random walks in confinement to geometry-controlled kinetics, Phys. Rep. 539, 225 (2014).

[59] A. Godec and R. Metzler, Universal proximity effect in target search kinetics in the few encounter limit, Phys. Rev. X 6, 041037 (2016).

[60] D. Grebenkov, R. Metzler, and G. Oshanin, Strong defocusing of molecular reaction times results from an interplay of geometry and reaction control, Comm. Chem. 1, 96 (2018); Full distribution of first exit times in the narrow escape problem, New J. Phys. 21, 122001 (2019); From singleparticle stochastic kinetics to macroscopic reaction rates: fastest first-passage time of $N$ random walkers, New J. Phys. 22, 103004 (2020).

[61] M. Kac, On distributions of certain Wiener functionals, Trans. Amer. Math. Soc. 65, 1 (1949).

[62] P. Erdös and M. Kac, On the number of positive sums of independent random variables, Bull. Amer. Math. Soc. 53, 1011 (1947).

[63] M. Abramowitz and I. A. Stegun, Handbook of Mathematical Functions, (New York: Dover, 1965).

[64] P. Lévy, Sur certains processus stochastiques homogènes (On certain homogeneous stochastic processes), Compositio Mathematica 7, 283 (1940).

[65] G. Oshanin, A. Mogutov and M. Moreau, Steady flux in a continuous-space Sinai chain, J. Stat. Phys. 73, 379 (1993).

[66] C. Monthus and A. Comtet, On the flux distribution in a one-dimensional disordered system, J. 
Phys. I. France 4, 635 (1994).

[67] G. Oshanin and G. Schehr, Two stock options at the races: Black-Scholes forecasts, Quant. Fin. 12, 1325 (2012).

[68] R. H. Cameron and W. T. Martin, Transformations of Wiener integrals under a general class of linear transformation, Trans. Amer. Math. Soc. 58, 184 (1945).

[69] R. H. Cameron and W. T. Martin, Evaluation of various Wiener integrals by use of certain SturmLiouville differential equations, Bull. Amer. Math. Soc. 51, 73 (1945).

[70] M. Kac, On distributions of certain Wiener functionals, Trans. Amer. Math. Soc. 65, 1 (1949).

[71] J. Ślȩzak, R. Metzler, and M. Magdziarz, Superstatistical generalised Langevin equation: nonGaussian viscoelastic anomalous diffusion, New J. Phys. 20, 023026 (2018).

[72] T. J. Lampo, S. Stylianidou, M. P. Backlund, P. A. Wiggins, and A. J. Spakowitz, Cytoplasmic RNA-Protein Particles Exhibit Non-Gaussian Subdiffusive Behavior, Biophys. J. 112, 532 (2017).

[73] D. S. Grebenkov, Time-averaged mean square displacement for switching diffusion, Phys. Rev. E 99, 032133 (2019). 